\title{
Fungal Diversity in World largest Man-made forest, Changa Manga, Pakistan
}

\author{
Abdul Rehman Niazi ${ }^{1 *}$, Najam-ul-Sehar Afshan ${ }^{2} \&$ Annum Razzaq ${ }^{1}$ \\ ${ }^{1}$ Department of Botany, University of the Punjab, Lahore, Pakistan \\ ${ }^{2}$ Centre for Undergraduate Studies, University of the Punjab, Lahore, Pakistan
}

\begin{abstract}
The Changa Manga is a first world largest artificial (man-made) forest and a wildlife park of Pakistan which was established in 1866. The forest is now maintained as national park and covers an area of 12,515 acres (226,000 ha). The forest is situated near Pattoki tehsil in district Kasur, Punjab, Pakistan. The forest has lower summer temperatures, less temperature fluctuation, higher humidity and milder winters. The average temperature of the forest is $75^{\circ} \mathrm{F}$ with $355 \mathrm{~mm}$ annual rainfall. These conditions are ideal for fungal flora to grow in these forests and allows a wide variety of fungi to flourish in the region.Many varieties of mushrooms are present on tree trunks and dungs with distinctive fruiting bodies. These fungi cover the area of wood rotting, decomposers, parasites as well as mycorrhizal. These fungi are important economically and they form often times obligatory relationship with vascular plants with access to key element for plant growth and forest product.37 species of ascomycota belonging to 30 genus and 12 families and 118 species of basidiomycota belonging to 62 genera and 22 families has been identified. During the survey different specimen of fungi were collected and to confirm their identity the internal transcribed spacer regions of the ribosomal DNA was sequenced and analyzed along with the morpho-anatomical characterization.
\end{abstract}

Key Words: Changa Manga, Fungal Diversity, Man-made forest,Pakistan

\section{Introduction}

The Changa Manga Forest, now maintained as a National Park, was planted on scrub-land in 1864 to supply fuel wood for locomotives running on the newly laid railroad from Peshawar to Karachi (Khan, 1962). The Changa Manga is a first world largest artificial (man-made) forest and a wildlife park of Pakistan. It was established in 1866 and named after two brothers Changa and manga. The Changa manga forest is present in the south $74 \mathrm{~km}$ (46miles) away of Lahore and $18 \mathrm{~km}$ (11miles) away from Phoolnagar. The forest is situated near Pattoki tehsil in district Kasur, Punjab, Pakistan. It falls under the irrigated plantation and today it covers a total area of 12,515 acres (226,000 ha) (Khan and Salman, 1969). Prominent tree species are Sheesham (DalbergiasissooRoxb.), mulberry (MorusalbaL.), babul (Acacia niloticaDelile.), different species of Eucalyptus L' Her and Populus L. which are the common tree species grown in the irrigated plantations. The weather of this forest is extreme during the months of May to July, with temperature $40-48{ }^{\circ} \mathrm{C}$. From the late June till August, the monsoon season starts with heavy rainfall throughout the province. Forest receive water from water canals and plays an important role in the weather of surrounding areas. Despite being in arid zone it reduces the temperature in neighborhood. These conditions are ideal for fungi to grow in this forest. Exploration of fungal diversity of man-made forest Changa manga ultimately lead associated with economically important plants and weeds (Biswas, 1987). Mushrooms are seasonal fungi, which occupy diverse niches in nature in the forest ecosystem. They predominantly occur during the rainy season and also during spring when the snow melts. This forest due to thick plantation and irrigation is the hot spot for number of fungi. These fungi cover the area of wood rotting, decomposers, parasites as well as mycorrhizal (Ahmed et al., 1997). Many of these fungi are associated mutualistically with the forest trees. These fungi are important economically and they form often times obligatory relationship with vascular plants with access to key element for plant growth and forest product (Read, 1998). There are total 164 species belonging to 97 genera and 36 families. This research work will be an attempt to explore the biodiversity of fungal flora of largest man-made Changa Manga forest, Pakistan. It would also be helpful in the up gradation of existing descriptions and illustrations of mycoflora, to explore economically important mushrooms and to characterize fungal taxa on morpho-anatomical and molecular basis to trace the phylogeny of these fungi. 


\section{Materials And Method}

Fresh specimens were morphologically characterized in the field. They were brought in laboratory and dried and vouchered. Free sections of the fungi were made and their microscopic features were observed. Measurements were taken with the help of micrometer and microscopic structures were sketched with camera lucida attached with the light microscope. Molecular analysis was done by using $2 \%$ CTAB method. The extracted DNA was amplified by using ITS1F fungal specific and ITS4 universal primer. The amplified PCR products were then sequenced by using same primer pairs from macrogen, Korea. The sequenced data were compared by using BLAST at NCBI data base. The closely related sequences were extracted and finally the phylogenetic analysis was performed by using MEGA.

\section{Results}

Following is the list of species of division Ascomycotina which are reported from Changa Manga.

\begin{tabular}{|c|c|c|c|c|c|c|}
\hline \multicolumn{2}{|c|}{ Family } & \multicolumn{2}{|c|}{ Genus } & \multicolumn{2}{|c|}{ Species } & \multirow{2}{*}{$\begin{array}{l}\text { Reference } \\
\text { Ahmad(1978) } \\
\text { Murakami(1993) }\end{array}$} \\
\hline 1 & Melanosporaceae & 1 & Chaetomiun & 1 & C.bostrychodes & \\
\hline 2 & Sordariaceae & 2 & Podospora & 2 & P. pauciseta & Ahmad (1978) \\
\hline \multirow[t]{2}{*}{3} & \multirow[t]{2}{*}{ Sphaeriaceae } & 3 & Thyridaria & 3 & T. cajugae & Ahmad(1962, 1969a) \\
\hline & & 4 & Valsaria & 4 & V. salvadornia & Ahmad(1956b, 1978) \\
\hline 4 & Comochaetaceae & 5 & Coniochaeta & 5 & C. velutina & Ahmad (1978) \\
\hline \multirow[t]{7}{*}{5} & \multirow[t]{7}{*}{ Xylariaceae } & 6 & Anthostomella & 6 & A.capparidis & Ahmad (1978) \\
\hline & & 7 & Daldinia & 7 & D. concentrica & $\begin{array}{l}\text { Ahmad(1956b, 1978) } \\
\text { Qureshi \& Jamal (1971) }\end{array}$ \\
\hline & & 8 & Hypoxylon & 8 & H.hypomiltum & Ahmad(1956b, 1978) \\
\hline & & 9 & Penzigia & 9 & P. capparidis & Ahmad(1956b, 1978) \\
\hline & & 10 & Rosellinia & 10 & R. aquita & Ahmad(1956b, 1978) \\
\hline & & \multirow[t]{2}{*}{11} & \multirow[t]{2}{*}{ Xylaria } & 11 & X. hypoxylon & $\begin{array}{l}\text { Ahmad (1969a, 1978) } \\
\text { Mirza\& Qureshi (1978) }\end{array}$ \\
\hline & & & & 12 & X. mali & \\
\hline 6 & Diatrypaceae & 12 & Hercospora & 13 & H. ahmadii & Ahmad (1972b) \\
\hline 7 & Hypocreaceae & 13 & Pseudonectriella & 14 & P. ahmadii & Ahmad (1969a, 1978) \\
\hline \multirow[t]{2}{*}{8} & \multirow[t]{2}{*}{ Pyronemaceae } & 14 & Coprotus & 15 & C.albidus & Ahmad (1978) \\
\hline & & 15 & Lasiobolus & 16 & L. pilosus & Mirza\& Qureshi (1978) \\
\hline \multirow[t]{3}{*}{9} & \multirow[t]{3}{*}{ Botryosphaeriaceae } & \multirow[t]{2}{*}{16} & \multirow[t]{2}{*}{ Botryosphaeria } & 17 & B.dothidea & Ahmad(1956b, 1972b,1978) \\
\hline & & & & 18 & B. plicatula & Ahmad (1978) \\
\hline & & 17 & Guignardia & 19 & G. cytisi & Ahmad (1978) \\
\hline \multirow[t]{2}{*}{10} & \multirow[t]{2}{*}{ Hysteriaceae } & 18 & Hysterographium & 20 & H.pleosporoides & Ahmad (1969a, 1978) \\
\hline & & 19 & Tryblidaria & 21 & T. pakistanica & Ahmad (1978) \\
\hline \multirow[t]{2}{*}{11} & \multirow[t]{2}{*}{ Mycosphaerellaceae } & 20 & Mycosphaerella & 22 & M. dalbergia & $\begin{array}{l}\text { Ahmad(1956b) Ahmad \& } \\
\text { Arshad(1972b) }\end{array}$ \\
\hline & & 21 & Preussia & 23 & P. disjuncta & Ahmad(1978) \\
\hline \multirow[t]{14}{*}{12} & \multirow[t]{14}{*}{ Pleosporaceae } & \multirow[t]{4}{*}{22} & Didymosphaeria & 24 & D. casurinae & Ahmad (1969a) \\
\hline & & & & 25 & D. culmicola & Ahmad $(1967,1969 a)$ \\
\hline & & & & 26 & D. oblitescens & Ahmad (1977) \\
\hline & & & & 27 & D. winteri & Ahmad $(1962,1969 a)$ \\
\hline & & \multirow[t]{2}{*}{23} & \multirow[t]{2}{*}{ Leptosphaeria } & 28 & L. abutilonis & Ahmad (1969a) \\
\hline & & & & 29 & L. asparagina & Ahmad(1978) \\
\hline & & \multirow[t]{2}{*}{24} & \multirow[t]{2}{*}{ Massaria } & 30 & M.epileuca & Ahmad(1956b) \\
\hline & & & & 31 & M. dubia & Ahmad(1964) \\
\hline & & 25 & Metasphaeria & 32 & M. ambigua & Ahmad(1956b) \\
\hline & & 26 & Paraphaeosphaeria & 33 & P. capparidicola & $\begin{array}{l}\text { Ahmad(1956b), Ghaffar\& } \\
\text { Abbas (1972) }\end{array}$ \\
\hline & & 27 & Phaeosphaeria & 34 & P. eustoma & Ahmad (1962) \\
\hline & & 28 & Pleospora & 35 & P. scrophulariaceae & $\begin{array}{l}\text { Ahmad \&Hussain (1971), } \\
\text { ahmad (1978) }\end{array}$ \\
\hline & & 29 & Strickeria & 36 & S. cephalandrae & Ahmad (1978) \\
\hline & & 30 & Trematosphaeria & 37 & T. britzelmayriana & Ahmad (1969a) \\
\hline
\end{tabular}


Following is the list of species of division Basidiomyotina reported from Changa Manga.

\begin{tabular}{|c|c|c|c|c|c|c|}
\hline 1 & $\begin{array}{l}\text { Hymenomycetes } \\
\text { (Sub-class) }\end{array}$ & 1 & Tremella & 1 & T. moriformis & Ahmad $(1956,1972)$ \\
\hline \multirow[t]{9}{*}{2} & \multirow[t]{9}{*}{ Phleogenaceae } & 2 & Phleogena & 2 & P. faginea & Ahmad (1972) \\
\hline & & \multirow[t]{5}{*}{3} & \multirow[t]{5}{*}{ Puccinia } & 3 & P. cacao & Ahmad (1956a,b) \\
\hline & & & & 4 & P. phyllocladiae & Ahmad (1956a,b) \\
\hline & & & & 5 & P. punctiformis & Ahmad (1956a,b) \\
\hline & & & & 6 & P. rufipes & Ahmad (1956a,b) \\
\hline & & & & 7 & P. kuehnii & Babar (2013) \\
\hline & & \multirow[t]{2}{*}{4} & \multirow[t]{2}{*}{ Ravenelia } & 8 & R. mimosae- himalayae & Ahmad (1956) \\
\hline & & & & 9 & R. sessilis & Ahmad (1956a,b) \\
\hline & & 5 & Uromyces & 10 & U. rumicis & Ahmad $(1956 a, b)$ \\
\hline \multirow[t]{2}{*}{3} & \multirow[t]{2}{*}{ Melampsoraceae } & 6 & Cerotelium & 11 & C.fici & $\begin{array}{l}\text { Ahmad (1956a,b) } \\
\text { Hasnain et al(1959) }\end{array}$ \\
\hline & & 7 & Uredo & 12 & U. dalbergia & Ahmad (1956a,b) \\
\hline 4 & Tilietiaceae & 8 & Tillietia & 13 & T. tumefaciens & $\begin{array}{l}\text { Ahmad (1956b,c) } \\
\text { Hasnain et al(1959) }\end{array}$ \\
\hline \multirow[t]{6}{*}{5} & \multirow[t]{6}{*}{ Corticiaceae } & 9 & Corticium & 14 & C.portentosum & Ahmad (1956b) \\
\hline & & 10 & Lopharia & 15 & L. fulva & Ahmad (1956b) \\
\hline & & \multirow[t]{2}{*}{11} & \multirow[t]{2}{*}{ Podoscypha } & 16 & P. parvula & $\begin{array}{l}\text { Ahmad } \\
1972 b)\end{array}$ \\
\hline & & & & 17 & P. petaloides & $\begin{array}{l}\text { Ahmad (1967, 1969, } \\
\text { 1972) }\end{array}$ \\
\hline & & \multirow[t]{2}{*}{12} & \multirow[t]{2}{*}{ Scytinostroma } & 18 & S. cystidiatum & Ahmad (1972a) \\
\hline & & & & 19 & S. portentosum & $\begin{array}{l}\text { Ahmad } \\
1972 b)\end{array}$ \\
\hline 6 & Clavariaceae & 13 & Lachnocladium & 20 & L. falvum & Ahmad (1956b) \\
\hline 7 & Ramariaceae & 14 & Pistillaria & 21 & P. filiformis & Ahmad (1972a) \\
\hline 8 & Hydnaceae & 15 & Mycorrhaphium & 22 & M. stereoides & $\begin{array}{l}\text { Ahmad } \\
1972 b)\end{array}$ \\
\hline \multirow[t]{20}{*}{9} & \multirow[t]{20}{*}{ Polyporarceae } & 16 & Aporium & 23 & A.carayae & $\begin{array}{l}\text { Ahmad \&ahmad } \\
(1982)\end{array}$ \\
\hline & & 17 & Bjerkandera & 24 & B.adusta & Ahmad (1972a) \\
\hline & & 18 & Ceriporia & 25 & C.ferruginicinata & $\begin{array}{l}\text { Ahmad \& Ahmad } \\
(1982)\end{array}$ \\
\hline & & & & 26 & C.leptoderma & $\begin{array}{l}\begin{array}{l}\text { Ahmad \& } \\
(1982)\end{array} \\
\end{array}$ \\
\hline & & 19 & Coriolus & 27 & C. hirrutus & $\begin{array}{l}\text { Ahmad (1972a), } \\
\text { Aoshima (1992) }\end{array}$ \\
\hline & & & & 28 & C. proteus & Ahmad (1972a) \\
\hline & & & & 29 & C. pubescens & Ahmad (1972a) \\
\hline & & 20 & Deadalea & 30 & D. flavida & $\begin{array}{l}\text { Ahmad } \\
(1956 b, 1972 b)\end{array}$ \\
\hline & & 21 & Ganoderma & 31 & G.ahmadii & Ahmad (1972a) \\
\hline & & 22 & Irpex & 32 & I.flavus & $\begin{array}{l}\text { Ahmad } \\
1972 b)\end{array}$ \\
\hline & & 23 & lenzites & 33 & L. platyphylla & $\begin{array}{l}\text { Ahmad(1967, 1969, } \\
1972)\end{array}$ \\
\hline & & 24 & phylloporus & 34 & P. bibulosa & Ahmad (1960a) \\
\hline & & \multirow[t]{3}{*}{25} & \multirow[t]{3}{*}{ Poria } & 35 & P. latemarginata & $\begin{array}{l}\begin{array}{l}\text { Qureshi } \quad \& \quad \text { Jamal } \\
(1971)\end{array} \\
\end{array}$ \\
\hline & & & & 36 & P. paradoxa & Ahmad (1972a) \\
\hline & & & & 37 & P. versipora & Ahmad $(1962,1969)$ \\
\hline & & \multirow[t]{2}{*}{26} & \multirow[t]{2}{*}{ Rigidoporus } & 38 & R. microporus & Ahmad (1980a) \\
\hline & & & & 39 & $R$. vinetus & $\begin{array}{l}\text { Ahmad \& Ahmad } \\
(1982)\end{array}$ \\
\hline & & \multirow[t]{3}{*}{27} & \multirow[t]{3}{*}{ Trametes } & 40 & T. cingulata & $\begin{array}{l}\text { Ahmad } \\
1972 b)\end{array}$ \\
\hline & & & & 41 & T. corrugata & $\begin{array}{l}\text { Ahmad (1967, 1969, } \\
1972)\end{array}$ \\
\hline & & & & 42 & T. ijubarksii & Ahmad \& Ahmad \\
\hline
\end{tabular}




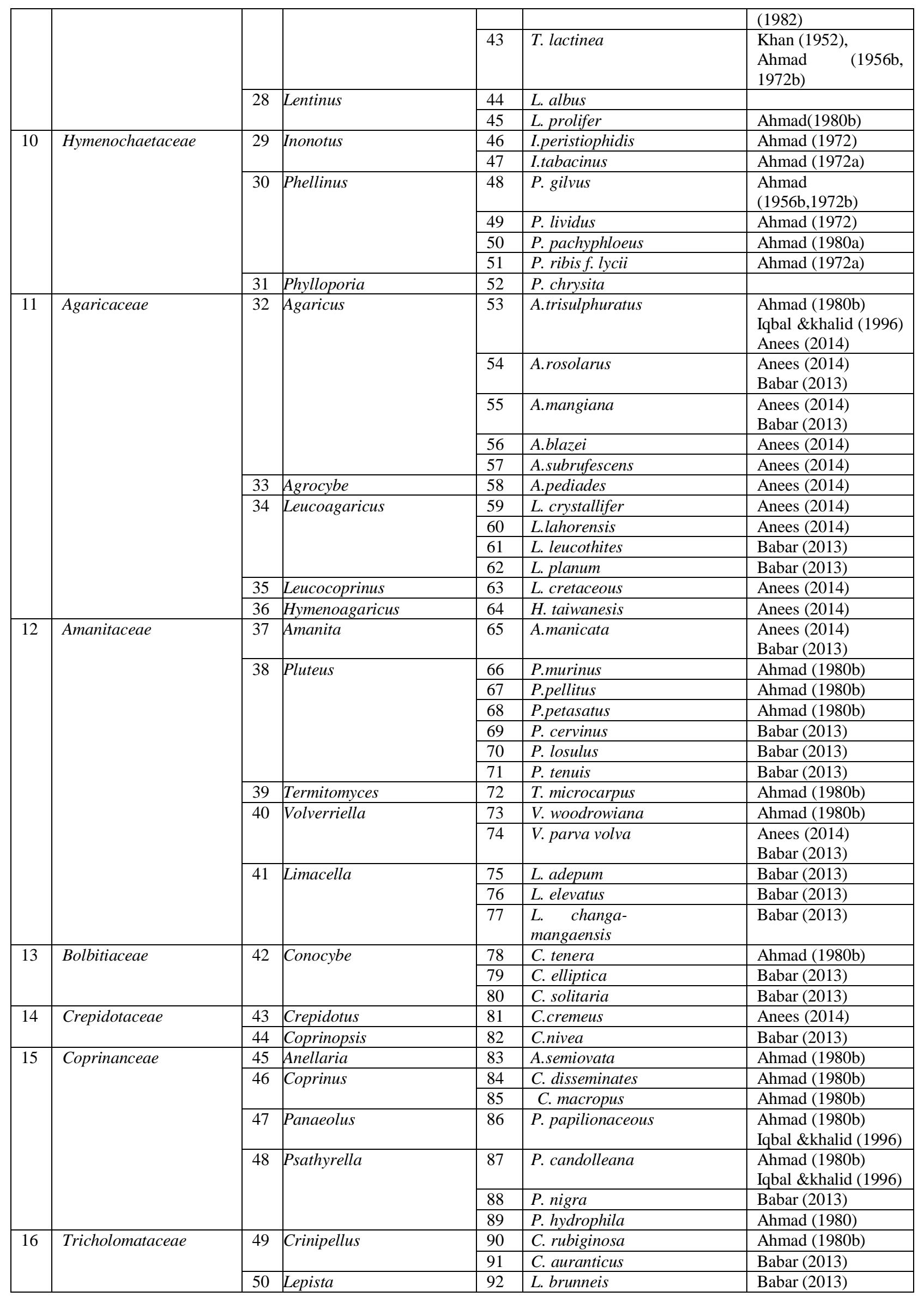




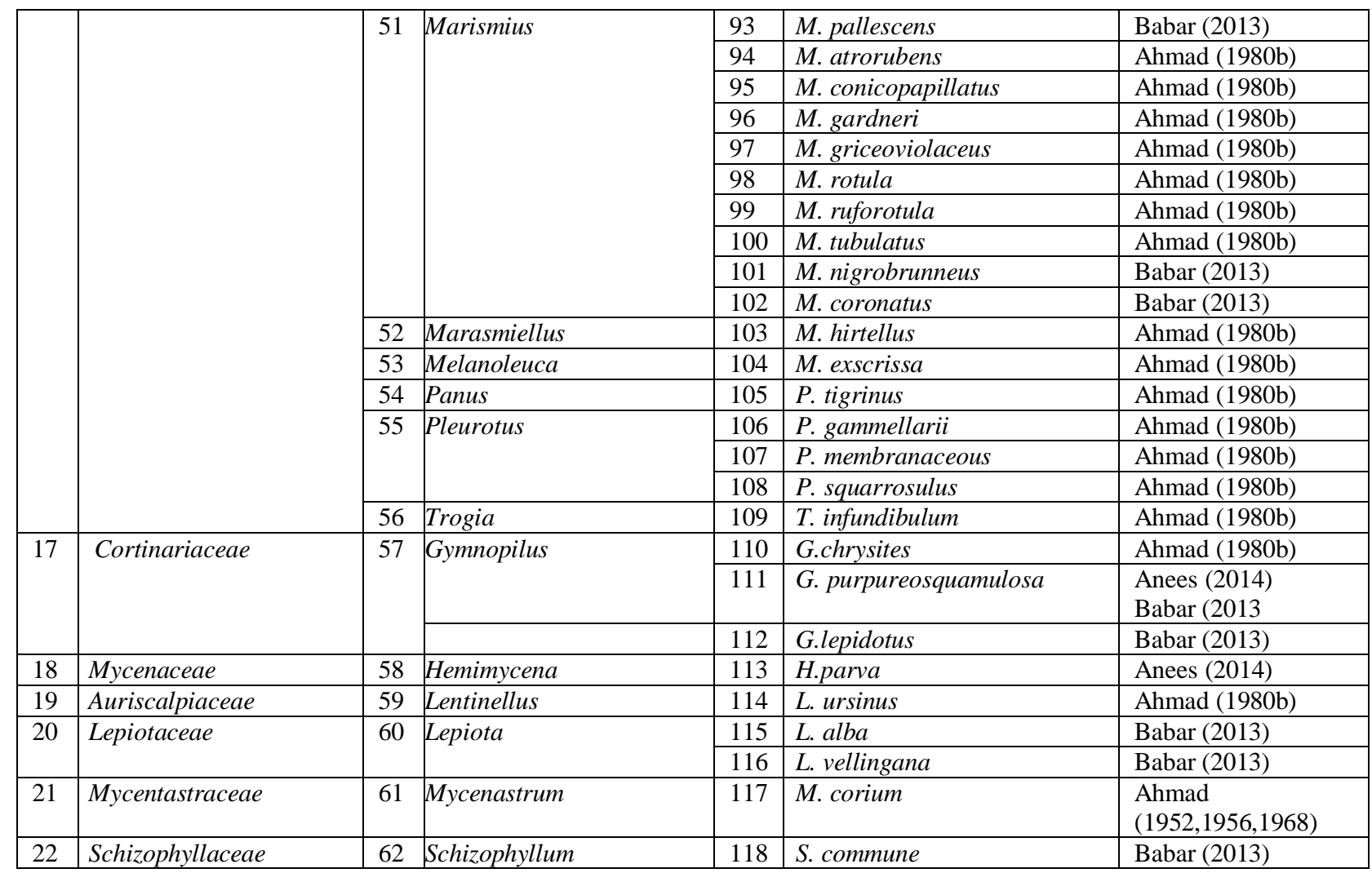

\section{Comments}

It is concluded that a much fungal diversity exist in this world largest man-made forest. There are more thorough surveys are needed to explore the maximum fungal diversity of this area. A number of species in this plantation waiting to be characterized and given named. Introduction of molecular techniques along with morphological and microscopic characterization seems to be quite effective up to the species level identification

\section{References}

[1] N. S. Afshan, A. N. Khalid, and A. R. Niazi, "New Records and distribution of rust fungi from Pakistan," Mycotaxon, vol. 105, pp. 257-267, 2008a.

[2] S. Ahmad,“Uredinales of Pakistan,"Biologia, vol. 2, pp. 29-101, 1956a.

[3] S. Ahmad, "Fungi of Pakistan, ” Biological Society of Pakistan, Lahore, Monograph. 1, 1956b,pp. 1-126.

[4] S. Ahmad, "Further contributions to the fungi of Pakistan I,"Biologia, vol. 6, pp. 117-136, 1960.

[5] S. Ahmad,"Further contributions to the fungi of Pakistan II,"Biologia, vol. 8, pp. 123-150, 1962.

[6] S. Ahmad, "Further contributions to the fungi of Pakistan IV,"Biologia, vol. 10, pp. 1- 62, 1964a.

[7] S.Ahmad,"Contribution to the fungi of Pakistan VI,"Biologia, vol. 13, pp. 15-42, 1967.

[8] S. Ahmad, "Fungi of Pakistan, ”Biological Society of Pakistan, Lahore, Monograph. 5, 1969a, Suppl. 1: 110.

[9] S.Ahmad, and M. Arshad, "Contribution to the fungi of West Pakistan XV,"Biologia, vol. 18, pp. 113-119.1972a.

[10] S. Ahmad, and M. Arshad,“Contribution to the fungi of West Pakistan XII,"Biologia, vol. 18, pp. 7-17, 1972b.

[11] S. Ahmad, "Contributions to the fungi of Pakistan XVIII,"Sultania, vol. 3, pp. 11-16, 1977.

[12] S. Ahmad, "Ascomycetes of Pakistan," Biological society of Pakistan, monograph 7 \& 8, 1978.

[13] S. Ahmad, "Contribution to the fungiof Pakistan XIX,"Bull. Mycologia, vol. 1 (1), pp. 27-32,1980a.

[14] S. Ahmad, "A contribution to the Agaricales of Pakistan,"Bull. Mycologia, vol. 1 (1), pp. 35-90,1980b.

[15] S. Ahmad, and N. Ahmad,"Contribution to the fungi of Pakistan XX,"Bull. Mycologia, vol. 2, pp. 79-86, 1982. 
[16] K. Aoshima, "List of some Pakitsani fungi," I. Polypores. In: Cryptogamic flora of Pakistan, T. Nakaike and S. Malik (eds.), vol. 1, pp. 139-144, 1992.

[17] A. Ghaffar, and S. Q. Abbas,“Fungi of Pakistan,” Suppl.II. Pak. Jour. Bot.4, 1972, pp. 195-208.

[18] A.N. Khalid, and S.H.Iqbal, "Additions to the rust flora of Pakistan," Pak. J. Bot., 28(1), pp. 115-117, $1996 a$.

[19] A. A. Khan, "Changa Manga Forest Plantation,” West Pakistan For. Rec., No. 6, vol. 10,1962.

[20] Khan, M. Aslam, and C. Salman, "Pakistan Medical Research Centre, Lahore,"Pakistan Journal of Zoology, vol. 1 (2), pp. 183-205,1969.

[21] J.H. Mirza, and M.S.A. Qureshi, "Fungi of Pakistan," University of Agri., Faisalabad, Pakistan (Memon).1978.

[22] Y. Murakami, "Larger fungi from Northern Pakistan," In: Crypt. Fl. Pak. Vol.2 (Eds. T. Nakaike and S. Malik), Nat. Sci. Mus. Tokyo, pp.105-147, 1993.

[23] M.A. Qureshi, and S.M. Jamal, "Fungi of hybrid poplar in West Pakistan,"Pak. Jour. Forestry, vol. 21, pp. 43$52,1971$.

[24] D. J. Read, "The mycorrhizal status of Pinus," In; Richardson D. M. (ed.), Ecology and biogeography of Pinus, Cambridge University Press, pp. 324-340,1998. 